\title{
AVALIAÇÃO DA IMPEDÂNCIA DE CHOQUE DA BLINDAGEM MULTICAMADA COM COMPÓSITO DE MATRIZ POLIÉSTER REFORÇA COM TECIDO DE JUTA*
}

\author{
Foluke Salgado de Assis ${ }^{1}$ \\ Sergio Neves Monteiro ${ }^{2}$ \\ Aderson Dionísio Leite Neto ${ }^{3}$ \\ Maria Clara Sagratzki Soares ${ }^{4}$ \\ Vinicius de Souza Pereira ${ }^{5}$
}

\section{Resumo}

Este trabalho visa apresentar alguns resultados obtidos através de ensaios balísticos realizados em sistemas de blindagem multicamada. Seguindo as especificações da norma americana NIJ 0101.06, foram realizados ensaios utilizando munição de calibre 7,62x $51 \mathrm{~mm}$, que possui velocidade acima de $800 \mathrm{~m} / \mathrm{s}$. A blindagem multicamada utilizada possui como camada frontal uma pastilha hexagonal cerâmica (alumina dopada com 4\% de nióbia), segunda camada de compósito de matriz poliéster reforçada com tecido de juta e como terceira camada uma placa de alumino 5052 H34. A utilização de compósitos poliméricos reforçados com fibras naturais em substituição às fibras de aramida é de grande interesse, pois além de desempenharem a mesma função no sistema de blindagem, possuem um valor de mercado bem menor. Com os resultados obtidos do ensaio balístico, os valores foram associados com o seu comportamento dinâmico utilizando o método do casamento de impedâncias.

Palavras-chave: Blindagem multicamada, fibra de juta, resina poliéster, comportamento dinâmico.

\section{ASSESSMENT SHOCK IMPEDANCE OF MULTILAYERED ARMOR WITH POLYESTER MATRIX COMPOSITES REINFORCED WITH JUTE CLOTH \\ Abstract}

This paper presents some results obtained through ballistic tests on Multilayered Armor System (MAS). Following the specifications of American standard NIJ 0101.06, the tests were realized with ammunition $7.62 \times 51 \mathrm{~mm}$ that has a velocity above of $800 \mathrm{~m} / \mathrm{s}$. The MAS is composed of a front layer a hexagonal ceramic chip (alumina doped with $4 \%$ of nióbia), the second layer of polyester matrix composite reinforced with jute cloth and the third layer an aluminum plate $5052 \mathrm{H} 34$. The utilization of polymeric composites reinforced with natural fibers to replace the aramid fibers is the big interest because they perform the same function in the armor system with the less expensive. The results obtained of ballistic tests were associated with their dynamic behavior, utilizing the method of impedances match.

Keywords: Multilayered armor, jute fiber, polyester resin, dynamic behavior.

1 Engenheiro Metalúrgico, mestre em Ciências dos Materiais, Instituto Militar de Engenharia, Rio de Janeiro, Rio de Janeiro - Brasil.

2 Engenheiro Metalúrgico, PhD em Materials Science And Engineering, Instituto Militar de Engenharia, Rio de Janeiro, Rio de Janeiro - Brasil.

3 Graduando em Engenharia, Instituto Militar de Engenharia, Rio de Janeiro, Rio de Janeiro - Brasil.

4 Graduanda em Engenharia, Instituto Militar de Engenharia, Rio de Janeiro, Rio de Janeiro - Brasil.

5 Graduando em Engenharia, Instituto Militar de Engenharia, Rio de Janeiro, Rio de Janeiro Brasil. 


\section{INTRODUÇÃO}

O aumento dos conflitos armados no Brasil, através de conflitos urbanos, e no mundo com guerras e atentados terroristas em conjunto das novas tecnologias de armas e munições impulsionam as pesquisas e desenvolvimentos por materiais que sejam capazes de resistir e proteger os seres humanos contra esses artefatos (1).

Os sistemas de blindagens multicamadas (MAS - Multilayered Armor System) proporcionam uma proteção pessoal leve, efetiva e têm como objetivo não apenas absorver a energia do projétil, mas também de impedir a penetração de fragmentos. Um sistema de blindagem multicamada típico é composto por uma placa cerâmica, seguida por camadas de fibras de aramida apoiadas em uma placa de alumínio (2). O material cerâmico que possui elevada resistência à compressão, elevada dureza e baixa resistência à tração tem como função dissipar a maior parte da energia de impacto através da deformação, erosão e fragmentação do projetil $(2,3,4)$. Na segunda camada, normalmente, são utilizados materiais sintéticos: Kevlar ${ }^{\circledR}$ (fibras de aramida) ou Dyneema ${ }^{\circledR}$ (polietileno de ultra-alto peso molecular) que possuem baixa densidade, elevada resistência à tração. Essa camada tem como principal objetivo dissipar a energia de impacto através da absorção da energia cinética dos fragmentos gerados pelo impacto do projetil com a cerâmica. Em alguns casos, uma terceira camada pode ser adicionada no sistema de blindagem multicamada que é composta por um metal dúctil que tem a função de restringir a penetração do projétil $(1,2)$. De acordo com a norma americana NIJ 0101.06 (5), o sistema de blindagem multicamada tem o nível de proteção classificado como III, isto é, consegue resistir ao impacto de munição perfurante com calibre 7,62 × 51 milímetros que possui velocidade de impacto superior a $800 \mathrm{~m} / \mathrm{s}$. O interesse em utilizar como segunda camada um material compósito de matriz polimérica reforçadas com fibras naturais como substituto das fibras sintéticas (fibras de Kevlar® ou Dyneema®) é pelo fato do material ter um custo menor, ser mais leve e tem o mesmo desempenho $(6,7,8)$. A utilização de compósitos reforçados com fibras naturais acarreta algumas vantagens, pois essas fibras são recursos alternativos, disponíveis em grande escala e possuem vantagens em relação às fibras sintéticas, tais como: biodegradabilidade, baixa densidade, natureza não tóxica, menor abrasividade nos equipamento que confeccionam os materiais, propriedades mecânicas aceitáveis e de baixo custo $(9,10)$. As fibras de juta são estudadas principalmente quando são utilizadas como material de reforço em matrizes poliméricas $(11,12)$. Este trabalho tem como objetivo associar o desempenho balístico com a impedância de choque do material testado como camada intermediária de compósitos de matriz poliéster reforçada com tecido de juta em comparação com a fibra de kevlar e outras fibras naturais.

\subsection{Comportamento dinâmico dos materiais}

O que define se um processo é estático ou dinâmico é a taxa da aplicação de uma força externa sobre o material. Para taxas de deformação lentas caracterizam um comportamento estático, ou seja, o fenômeno de carregamento é experimentado por todo o material. Já para taxas de deformação elevadas, obtém-se um comportamento dinâmico, onde o carregamento torna-se um fenômeno localizado que se propaga no material como uma onda de tensão. O impacto de um projétil em um material resulta em altas taxas de deformação e essa resposta dinâmica pode ser considerada como comportamento balístico do material. Dependendo da 
resposta do material à solicitação externa, as ondas geradas podem ser dadas na forma de ondas elástica, plástica e de choque (13).

\subsection{Interação e reflexão das ondas de choque}

O sistema de blindagem multicamada utiliza diferentes materiais que propiciam desempenho e leveza. Com a união desses materiais são criadas interfaces entre os mesmo e a interação da onda de choque nessas interfaces varia de material para material, e estes comportamentos podem ser analisados pelo método gráfico (pressão versus velocidade da partícula) do casamento de impedância. Quando a onda de choque passa pela interface entre dois materiais, uma pressão $(P)$ é formada para certa velocidade de partícula $U_{p}$ e pode ser medida através do parâmetro de impedância de choque (Z), que é definido como o produto da densidade $\left(\rho_{0}\right)$ e velocidade da onda de choque $\left(U_{s}\right)$ do material, caso o valor $U_{s}$ seja desconhecido, pode-se utilizar no seu lugar a velocidade sônica da onda $\left(\mathrm{C}_{0}\right)$. Há dois casos que se deve levar em questão quanto ao estudo de casamento de impedâncias (13):

A) Transferência de uma onda de choque de um material A (baixa impedância) para um material B (alta impedância):

Este tipo de transferência ocorre em um material A (camada externa) ser menos denso que o material B (camada intermediaria). Na FIG. 1(a) é mostrada a variação da pressão em função da velocidade de partícula para os materiais $A$ e $B$. A inclinação da linha pontilhada na pressão $P_{1}$ é a impedância de choque e explica a descontinuidade na pressão (ou densidade) durante o impacto e a curva AR representa a inversão da curva $A$, sendo o ponto $\left(U_{p 1}, P_{1}\right)$ comum entre as curvas AR e $A$ e o ponto $\left(U_{p 2}, P_{2}\right)$ sendo comum entre as curvas $A R$ e B. Na FIG. 1(b) são mostrados os perfis de pressão ao atingir a interface e pode-se observar que para manter o equilíbrio entre as pressões, a pressão $P_{1}$ da frente de choque aumenta descontinuamente para $\mathrm{P}_{2}$. A partir deste momento, a onda de choque é decomposta em duas ondas: uma se propagando no material $A$ (no sentido oposto) e outra se propagando no material $\mathrm{B}$. Em t3 e t4 à frente de pressão $\mathrm{P}_{1}$ encontra a onda de choque remanescente e cai para $\mathrm{P}_{2}-\mathrm{P}_{1}$ (onda compressiva). A velocidade de partícula $U_{\mathrm{p} 2}$ se mantém a mesma na região de alta pressão, em $A$ e $B$, sendo assegurada à continuidade de pressão e velocidade da partícula [13].
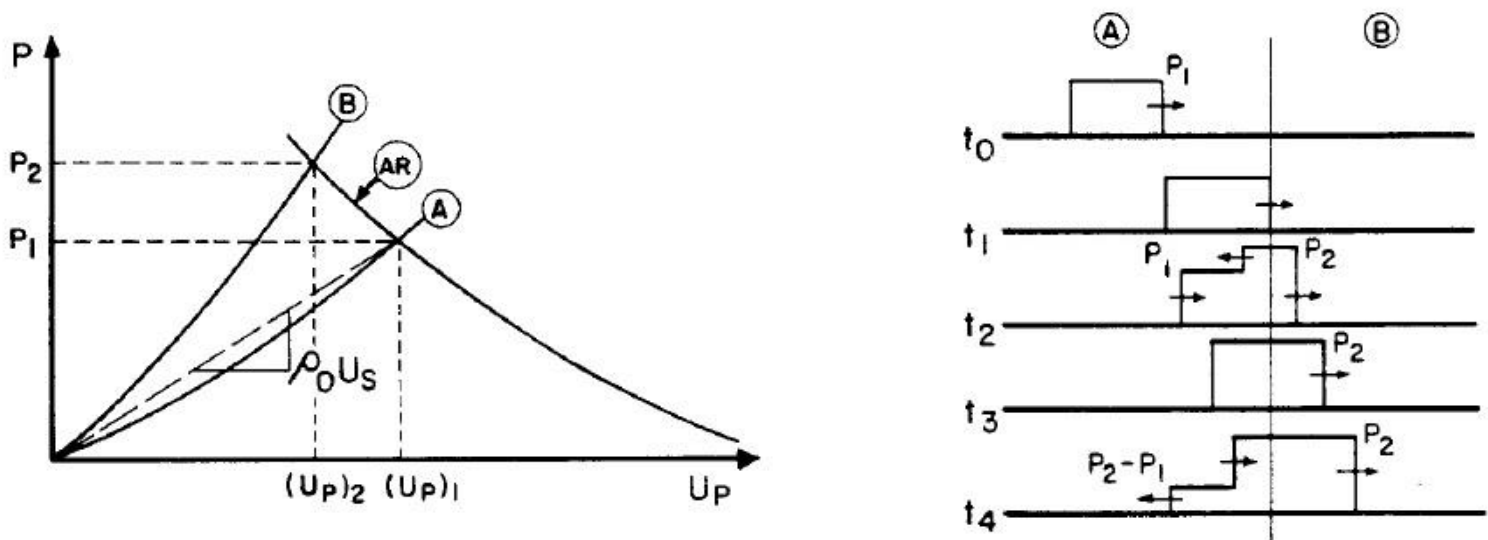

Figura 1 - Transmissão da onda de choque de um meio de baixa impedância para um meio de alta impedância de choque: (a) Gráfico pressão versus velocidade da partícula; (b) Perfil de pressão (7). 
B) Transferência de uma onda de choque de um material A (alta impedância) para um material B (baixa impedância):

É a situação inversa do caso (a), isto é, o material A (camada externa) ser mais denso que o material $B$ (camada intermediária), como é mostrado na Figura 2. Neste caso, a pressão $\mathrm{P}_{1}$ é maior que a pressão $\mathrm{P}_{2}$ e no tempo $\mathrm{t}_{4}$ (Figura. 2.a), um pulso trativo será formado, propagando-se em ambas as direções, ou seja, tanto para dentro do material $B$ como para o material $A$. Caso essa onda trativa tenha uma amplitude suficientemente alta, ocorrerá estilhaçamento do material (13).
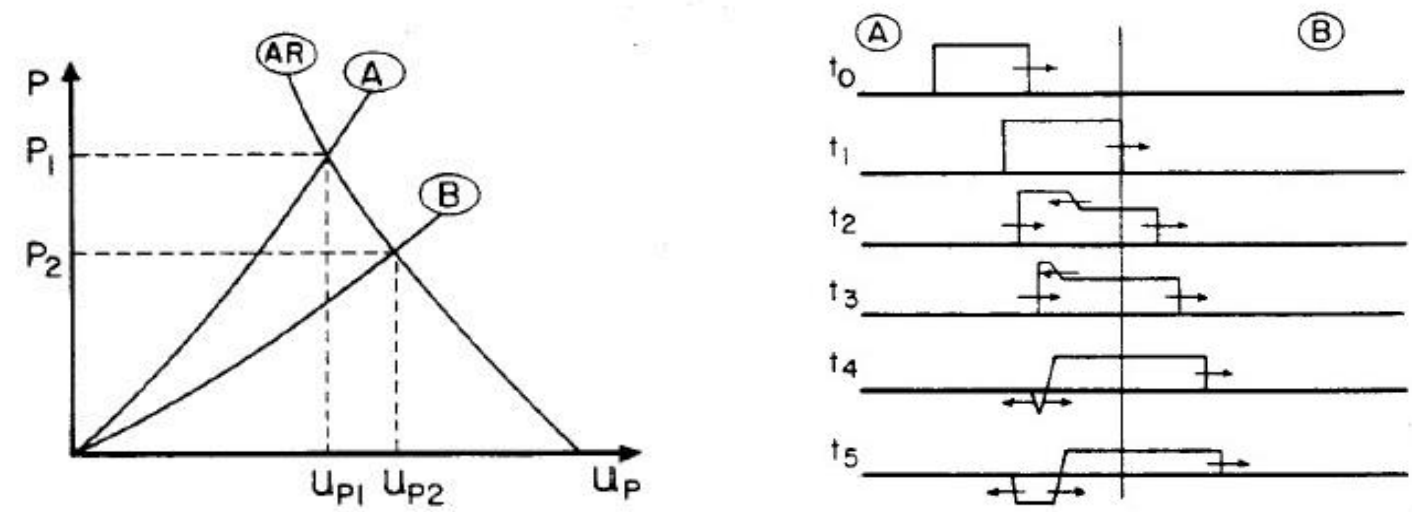

Figura 2 - Transferência da onda de choque de um meio com alta impedância para um meio com baixa impedância: (a) Gráfico pressão versus velocidade de partícula; (b) perfil de pressão (13).

\section{MATERIAIS E MÉTODOS}

O material cerâmico, primeira camada do sistema de blindagem, utilizado foi alumina $\left(\mathrm{Al}_{2} \mathrm{O}_{3}\right)$ dopada com nióbia $\left(\mathrm{Nb}_{2} \mathrm{O}_{5}\right)$ na proporção de $4 \%$ em massa, com geometria hexagonal. Já a segunda camada é formada pela placa de material compósito, que foi fabricada com reforço de tecido de juta. As placas foram confeccionadas em matriz de poliéster, na proporção de $30 \%$ em volume de tecido. A terceira camada foi composta por uma placa de alumínio 5052 H34.

A fabricação das pastilhas cerâmicas se deu da seguinte forma: a alumina, a nióbia e O PEG (polietileno glicol) foram devidamente pesados e em seguidas acondicionado num moinho de bolas de alumina por 12 horas. Ao final desse processo, o material foi levado e deixado em uma estufa a $80^{\circ} \mathrm{C}$ para que ocorresse a secagem, no período de 48 horas. Após essa etapa ocorreu às etapas de maceração e peneiramento para obtenção do pó, em seguida ocorreu à prensagem sob uma pressão de 60 MPA. Com isso, foram obtidos os corpos verdes e para finalizar a confecção das pastilhas cerâmicas, estes corpos foram levados ao forno para que fossem sinterizados.

Os corpos de prova dos compósitos foram confeccionados em uma matriz metálica retangular com dimensões $15,0 \mathrm{~cm} \times 12,8 \mathrm{~cm}$, objetivando uma espessura final da placa de $1,0 \mathrm{~cm}$. O tecido de juta foi cortado de acordo com o espaço interno da matriz e misturado com a resina poliéster. $O$ preparo da resina foi realizado por meio da mistura com o catalizador peróxido metil-etil-cetona, numa proporção de $1 \%$ em peso. Após a mistura do tecido com a resina poliéster dentro da matriz metálica, a mesma foi submetida à pressão de 5 toneladas durante o tempo de cura, que foi de 24 horas. Na Figura 4 é mostrado o sistema de blindagem multicamada utilizado neste trabalho. Foram confeccionados no total de 10 corpos de prova para os ensaios balísticos. 


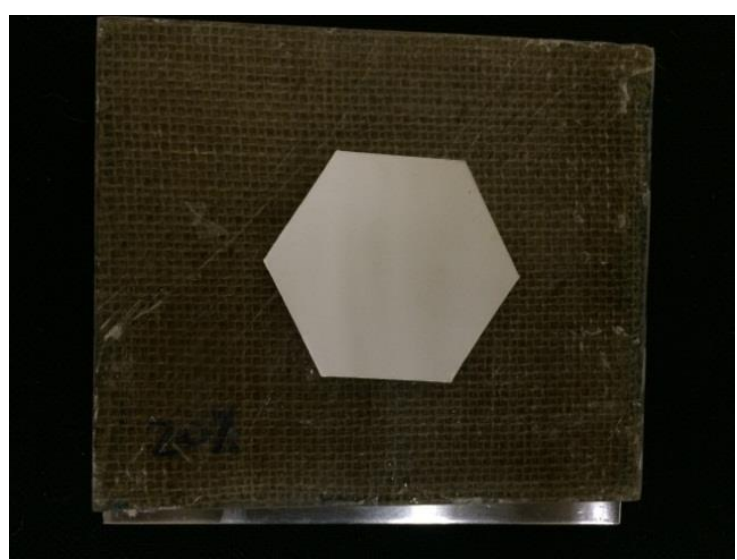

Figura 2 - Blindagem multicamada tendo como camada intermediária o compósito de matriz poliéster reforçado com tecido de juta

Os ensaios balísticos foram realizados no Campo de Prova da Marambaia - RJ, na linha IV de ensaios para armas leves. Para estes ensaios foi utilizado um provete com calibre 7,62 mm dotado de mira laser, uma barreira óptica modelo B471 da HIP, um radar WEIBEL DOPPER, dispositivo de fixação do alvo e munição calibre 7,62 x $51 \mathrm{~mm}$ comum. Para o calculo da onda e impedância de choque, utilizados no método de casamento de impedância, foram utilizados equações de conservação de momento de Rankine-Hugoniot e Estado do Material.

1) Momento: $\left(P-P_{0}\right)=\rho_{0} U_{S} U_{P}$

2) Estado do Material: $U_{s}=C_{0}+S_{1} U_{p}$

Onde: velocidade da partícula $\left(U_{P}\right)$, velocidade de choque $\left(U_{S}\right)$, densidade $(\rho)$, pressão $(P)$, velocidade do som do material a pressão igual a zero $\left(\mathrm{C}_{0}\right)$, S é um parâmetro empírico.

\section{RESULTADOS E DISCUSSÃO}

Os parâmetros característicos dos materiais utilizados nesses cálculos são mostrados na Tabelas 1 e 2. Não foi possível obter, na literatura, os parâmetros específicos para o compósito poliéster-juta, então, foi utilizada a velocidade da onda elástica $\left(\mathrm{C}_{0}\right)$, no lugar da velocidade de onda de choque $\left(U_{s}\right)$. Para a resina poliéster e para fibra de juta, foram encontrados, na literatura, valores mínimos e máximos referente ao módulo de elasticidade $(9,10)$.

Tabela 1. Valares da velocidade da onda de choque e dos parametros característicos de cada material

\begin{tabular}{cccccc}
\hline Material & $\boldsymbol{\rho}\left(\mathbf{k g} / \mathbf{m}^{3}\right)$ & $\mathbf{C}(\mathbf{m} / \mathbf{s})$ & $\mathbf{S}$ & $\mathbf{U}_{\mathbf{s}}(\mathbf{m} / \mathbf{s})$ & Referência \\
\hline Chumbo & 11300 & 2066 & 1,517 & $2066+1,517 U_{p}$ & $(13)$ \\
Aramida & 1440 & 1180 & 2,420 & $1180+2,420 U_{p}$ & $(13)$ \\
Alumínio & 2700 & 5328 & 1,338 & $5328+1,338 U_{\mathrm{p}}$ & $(13)$ \\
Alumina & 3530 & & & 10375,39 & $(7)$ \\
\hline
\end{tabular}


Tabela 2. Valores do módulo de elasticidade e da onda elástica do compósito pooliéster-juta

\begin{tabular}{cccccc}
\hline Material & $\begin{array}{c}\boldsymbol{\rho} \\
\left(\mathbf{g} / \mathbf{c m}^{3}\right)\end{array}$ & $\begin{array}{c}\mathbf{E}_{\text {min. }} \\
(\mathbf{G P a})\end{array}$ & $\begin{array}{c}\mathbf{E}_{\text {máx. }} \\
(\mathbf{G P a})\end{array}$ & $\begin{array}{c}\mathbf{C}_{\mathbf{0}(\min .)} \\
(\mathbf{m} / \mathbf{s})\end{array}$ & $\begin{array}{c}\mathbf{C}_{\text {o(máx.) }} \\
(\mathbf{m} / \mathbf{s})\end{array}$ \\
\hline Juta & 1,30 & 13,00 & 27,00 & - & - \\
Poliéster & 1,10 & 2,06 & 4,46 & - & - \\
Compósito Poliéster-Juta $(30 \%)$ & 1,16 & 5,34 & 11,19 & 2145,97 & 3105,47 \\
\hline
\end{tabular}

O gráfico do método de casamento de impedância é mostrado na FIG. 4 analisando a interação das ondas de choque em cada interface do sistema de blindagem multicamada. Neste gráfico foi utilizado o sistema de blindagem multicamada utilizando como camada intermediária o compósito de matriz poliéster reforçado com $30 \%$ de fibras de juta. A resolução dos cálculos nas interfaces pode ser resumida em três etapas: interface projétil (chumbo)/alumina; interface alumina/compósito; e interface compósito/alumínio. Também foram consideradas as interfaces alumina/aramida e aramida/alumínio para fins comparativos. A velocidade de projétil adotada 863,63.

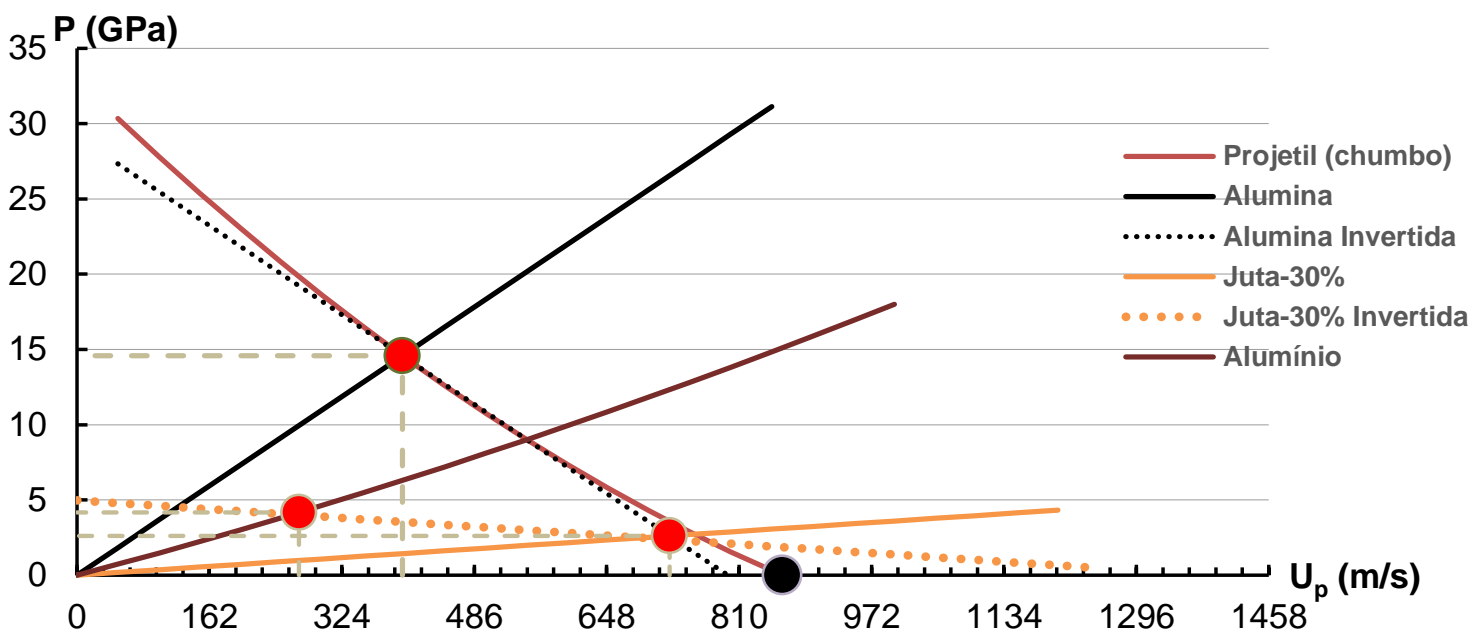

Os valores encontrados para interação das ondas de choque, nas interfaces dos sistemas de blindagem multicamada, estudada neste trabalho, utilizando o método de casamento de impedância, são mostrados na Tabela 3. Na interface alumina/compósito e alumina/aramida a onda refletiva é de natureza trativa, pois a alumina possui uma impedância maior que os materiais utilizados como segunda camada (tanto compósito quanto aramida), ocasionando o seu estilhaçamento, como previsto pelo método do casamento de impedâncias.

Tabela 3.Valores da velocidade de partícula, velocidade de onda de choque e pressão nas interfaces de cada componente da blindagem multicamada e a natureza da onda de choque.

\begin{tabular}{cccccc}
\hline Interfaces & & $\mathbf{U}_{\mathbf{p}}(\mathbf{m} / \mathbf{s})$ & $\begin{array}{c}\text { Pressão } \\
\text { (GPa) }\end{array}$ & $\mathbf{U}_{\mathbf{s}}(\mathbf{m} / \mathbf{s})$ & $\begin{array}{c}\text { Natureza da onda } \\
\text { de choque }\end{array}$ \\
\hline Chumbo/Alumina & & 398,14 & 14,58 & 10375,35 & Compressiva \\
& Mín. & 745,6 & 1,86 & 2145,97 & Trativa \\
Alumina/Poliéster-juta (30\%) & Máx. & 724,97 & 2,61 & 3105,47 & Trativa \\
& Mín. & 202,13 & 3,06 & 5598,45 & Compressiva \\
Poliéster-juta (30\%)/Alumínio & Máx. & 271,47 & 4,17 & 5691,23 & Compressiva \\
Alumina/Aramida & & 714,55 & 2,99 & 2909,211 & Trativa \\
Aramida/Alumínio & & 365,84 & 5,75 & 5817,49 & Compressiva \\
\hline
\end{tabular}


Os resultados obtidos no ensaio balístico são mostrados na Tabela 4, destacando-se os diferentes materiais utilizados como camada intermediária.

Tabela 4. Médias dos valores obtidos nos ensaios balísticos.

\begin{tabular}{ccccccc}
\hline $\begin{array}{c}\text { Camada } \\
\text { Intermediária }\end{array}$ & $\begin{array}{c}\text { Espessura } \\
\text { Média } \\
(\mathbf{m m})\end{array}$ & $\begin{array}{c}\mathbf{\rho} \text { média } \\
\left(\mathbf{g} / \mathbf{c m}^{3}\right)\end{array}$ & $\begin{array}{c}\text { Indentação } \\
\text { média }(\mathbf{m m})\end{array}$ & $\begin{array}{c}\text { Velocidade de } \\
\text { Impacto Média } \\
(\mathbf{m} / \mathbf{s})\end{array}$ & $\begin{array}{c}\text { Energia de } \\
\text { Impacto Média } \\
(\mathbf{k J})\end{array}$ & Referências \\
\hline $\begin{array}{c}\text { Poliéster-Juta } \\
(30 \%)\end{array}$ & 10,61 & 1,17 & 17,88 & 853,08 & 3,53 & ${ }^{*} \mathrm{PT}$ \\
$\begin{array}{c}\text { Epóxi-sisal } \\
(30 \%)\end{array}$ & 11,73 & 1,14 & 16,03 & 827,95 & 3,32 & $(6)$ \\
$\begin{array}{c}\text { Epóxi-Juta } \\
(30 \%)\end{array}$ & 11,42 & 1,17 & 20,67 & 833,81 & 3,37 & $(8)$ \\
Aramida & 10 & 1,40 & 22,67 & 836,81 & 3,41 & $(8)$ \\
\hline
\end{tabular}

As impedâncias de choque de cada componente da blindagem multicamada são mostradas na Tabela 5. De acordo com os resultados expressos nessa tabela, a aramida exibiu o maior valor de impedância de choque dentre os materiais utilizados como camada intermediária. Esse valor justifica a menor redução de energia de impacto, verifica pela maior profundidade média de indentação $(22,67 \mathrm{~mm})$ obtida em trabalhos anteriores, pois, em princípio, para uma maior impedância de choque da camada intermediaria, maior a energia do pulso de compressão transmitida (8).

Tabela 5. Valores da Impedância de choque dos componentes da blindagem multicamada.

\begin{tabular}{ccccc} 
Material & $\begin{array}{c}\text { Densidade } \\
\left(\mathbf{k g} / \mathbf{m}^{3}\right)\end{array}$ & $\begin{array}{c}\text { Velocidade de } \\
\text { onda }(\mathbf{m} / \mathbf{s})\end{array}$ & $\begin{array}{c}\text { Impedância } \\
\left(\mathbf{k g} /\left(\mathbf{m}^{2} . \mathbf{s}\right) \mathbf{)}\right.\end{array}$ & Referência \\
\hline Poliéster-juta (30\%) (mín.) & 1160 & 2145,97 & $2,49 \times 10^{6}$ & ${ }^{*} \mathrm{PT}$ \\
Poliéster-juta (30\%) (máx.) & 1160 & 3105,47 & $3,60 \times 10^{6}$ & ${ }^{*} \mathrm{PT}$ \\
Chumbo & 11300 & $2.066,00$ & $23,35 \times 10^{6}$ & ${ }^{*} \mathrm{PT}$ \\
Alumínio & 2700 & $5.817,49$ & $15,85 \times 10^{6}$ & ${ }^{*} \mathrm{PT}$ \\
Aramida & 1440 & $2.909,21$ & $4,19 \times 10^{6}$ & ${ }^{*} \mathrm{PT}$ \\
Epóxi-juta (mín.) & 1167 & $2.188,03$ & $2,55 \times 10^{6}$ & {$[8]$} \\
Epóxi-juta (máx.) & 1167 & $2.895,94$ & $3,38 \times 10^{6}$ & {$[8]$} \\
Epóxi-sisal (mín.) & 1155 & $1.948,91$ & $2,25 \times 10^{6}$ & {$[6]$} \\
Epóxi-sisal (máx.) & 1155 & $2.955,22$ & $3,41 \times 10^{6}$ & {$[6]$} \\
Alumina & 3530 & $10.375,39$ & $36,62 \times 10^{6}$ & {$[7]$} \\
\hline
\end{tabular}

\section{CONCLUSÃO}

Nenhum dos corpos de prova, testados, foi perfurado pelo projétil de calibre $7,62 \times$ $51 \mathrm{~mm}$. Pelo fato da alumina possuir maior valor de impedância, $36,62 \times 10^{6} \mathrm{~kg} / \mathrm{m}^{2} \mathrm{~s}$, em comparação com todos os materiais estudados, independente da camada intermediária utilizada na composição da blindagem multicamada, a onda refletida será de natureza trativa, proporcionando o estilhaçamento da camada cerâmica durante os ensaios balísticos.

Dentre os materiais utilizados como camada intermediária o tecido de aramida exibiu maior impedância, $4,19 \times 10^{6} \mathrm{~kg} / \mathrm{m}^{2} \mathrm{~s}$. Esse valor mais elevado justifica a menor redução de energia de impacto, e consequentemente menor eficiência balística da blindagem multicamada que utiliza o tecido de aramida como camada intermediária. 


\section{Agradecimentos}

A CAPES pelo incentivo financeiro.

\section{REFERÊNCIAS}

1. WANG, L.; KANESALINGAM, S.; NAYAK, R.; PADHYE, R. Recent trends in Ballistic Protection. Textiles and Light Industrial Science and Technology (TLIST), Vol. 3. P. 3747. 2014.

2. MONTEIRO, S.N.; LIMA JR, E.P.; LOURO, L.H.L., SILVA, L.C.; DRELICH, J.W. Unlocking function of aramida fibers in multilayered ballistic armor. Accept for publication un Metallurgical and Materials Transactions A, November, 2014.

3. MEDVEDOVSKI, E. Ballistic Performance of Armour Ceramics: Influence of Design and Structure. Part 1. Ceramics International. Vol. 36, p. 2103-2115. 2010.

4. DA SILVA, M.V.; STAINER, D.; AL-QURESHI, H.A.; HOTZA, D. Blindagens Cerâmicas para Aplicações Balísticas: Uma Revisão. Cerâmica, Vol. 60, p. 323-331. 2014.

5. NIJ Standards-0101.06.Ballistic Resistance of Body Armor.U.S. Department of Justice/Office of Justice Programs - National Institute of Justice. 2008.

6. ARAÚJO, B.M. Avaliação do Comportamento Balístico de Blindagem Multicamada com Compósito de Epóxi Reforçado com Fibra de Sisal. Dissertação de Mestrado do Curso de Pós-Graduação em Ciência dos Materiais no Instituto Militar de Engenharia. 2015.

7. DA SILVA, L.C. Comportamento Balístico de Compósitos Epóxi-Fibra Natural em Blindagem Multicamada. Tese de Doutorado do Curso de Pós-Graduação em Ciência dos Materiais no Instituto Militar de Engenharia. 2014b.

8. DA LUZ, F.S. Avaliação do Comportamento Balístico de Blindagem Multicamada dom Compósito de Epóxi Reforçado com Fibra de Juta. Dissertação de Mestrado do Curso de Pós-Graduação em Ciência dos Materiais no Instituto Militar de Engenharia. 2014.

9. SATYANARAYANA, K.G.; GUIMARÃES, J.L.; WYPYCH, F. Studies on lignocellulosic fibers of Brazil. Part I: Source, production, morphology, properties and applications. Composites: Part A, v. 38, p. 1694-1709, 2007.

10. MONTEIRO, S.N.; LOPES, F.P.D.; BARBOSA, A.P.B.; BEVITORI, A.B.; DA SILVA, I.L.A.; DA COSTA, L.L. Natural Lignocelullosic Fibers as Engineering Materials - An Overview. Metallurgical and Materials Transactions A, Vol. 42A, p. 2963-2974. 2011.

11. DA SILVA, I. L. A. Propriedades e Estruturas de Compósitos Poliméricos Reforçados com Fibras Contínuas de Juta. Tese de Doutorado do Curso de Pós-Graduação em Engenharia e Ciências de Materiais na Universidade Estadual do Norte Fluminense. 2014c.

12. WAMBUA, P.; VANGRIMDE, B.; LOMOV, S.; VERPOEST, I. The Response of Natural Fibre Composites to Ballistic Impact by Fragment Simulating Projectiles. Composite Structures, Vol. 77, p. 232-240. 2007.

13. MEYERS, M.A. Dynamic behavior of materials. New York: John Wiley \& Sons, 1994. 